\title{
Publisher Correction: Democratic classification of free-format survey responses with a network-based framework
}

Tatsuro Kawamoto (iD) and Takaaki Aoki (iD

Correction to: Nature Machine Intelligence https://doi.org/10.1038/s42256-019-0071-y, published online 9 July 2019.

In the first correction to this Article, where it indicated that $\sigma$ should have been $\sigma_{i}$, in fact, it should have been $\left\{\sigma_{i}\right\}$. The Article has been corrected accordingly.

Published online: 30 July 2019

https://doi.org/10.1038/s42256-019-0090-8 